\title{
Saúde mental em estudantes de medicina
}

\section{Mental health in medical students}

\author{
Júlio Aragão***; Bruna Casiraghi* **; Érica Mota*; Mariana Abrahão*; Tiago Almeida*; Ana Baylão*; Pedro Araújo* \\ *Centro Universitário de Volta Redonda, **Universidade do Minho
}

\begin{abstract}
Resumo
Estudantes de medicina constituem um grupo muito suscetível ao desenvolvimento de Transtornos Mentais Comuns (TMC). A necessidade de acompanhamento dos estudantes de medicina motivou um levantamento em um curso de Medicina. Utilizou-se o GHQ-12. Dos 631 alunos obteve-se $67,82 \%$ (428) de cobertura. A prevalência de TMC atingiu 58,8\% dos discentes, com maior ocorrência no terceiro ano e nos últimos dois anos. Os resultados demonstram a necessidade de suporte acadêmico, para evitar desdobramentos graves e danosos à saúde mental, como também para uma melhora no acolhimento exercido aos seus futuros pacientes, ampliando a qualidade e humanizando a medicina.

Palavras chave: Saúde mental, estudantes de medicina, depressão, ansiedade
\end{abstract}

\begin{abstract}
Medical students constitute a group very susceptible to the development of Common Mental Disorders (CMD). The need for monitoring of medical students led a study in a medical school. GHQ-12 was used. Of the 631 students, $67.82 \%$ (428) of coverage were obtained. The prevalence of CMD reached $58.8 \%$ of students with greater occurrence in the third year and in the last two years. The results demonstrate the need for academic support, to avoid serious and harmful consequences to mental health, as well as to an improvement in the care given to their future patients, increasing quality and humanizing medicine ..

Keywords: Mental health, medical students, depression, anxiety
\end{abstract}

\section{Introdução}

Estudantes de medicina são reconhecidos por enfrentarem um curso desgastante e repleto de situações potencialmente estressantes. Expectativas e responsabilidades aumentam progressivamente durante o curso, agregando tensões e angústias que afligem significativamente a saúde destes. Nesse contexto árduo, a saúde mental talvez seja a parte mais afetada dos futuros médicos (Alves, 2014; Andrade et al., 2014; Ferreira, Kluthcovsky, \& Cordeiro, 2016; Tanaka, Furlan, Branco, \& Valerio, 2016).

A Organização Mundial da Saúde define saúde mental como um estado de bem estar em que o indivíduo é capaz de realizar seus potenciais, lidar com estresses cotidianos e trabalhar prolificamente, estando inserido dentro do amplo conceito de saúde (World Health Organization,
Victorian Health Promotion Foundation, \& University of Melbourne, 2004). Os TMC são caracterizados por sintomas psiquiátricos não psicóticos insuficientes para concluir diagnóstico formal, porém que comprometem o desempenho de atividades diárias (Pereira, Padoim, \& Junior, 2014). Destacam-se sintomas como irritabilidade, dificuldade de concentração e fadiga. A falta de traquejo em lidar com estressores e a negligência à presença de TMC podem ocasionar desdobramentos ainda mais sérios e debilitantes para a saúde mental e performance do estudante de medicina (Cunha et al., 2009; Rocha \& Sassi, 2013; Santa \& Cantilino, 2016).

Conflitos de adaptação no início do curso, progressiva conscientização a respeito das dificuldades da carreira, pressão para assimilar grandes conteúdos e falta de tempo para atividades sociais contribuem para o desencadeamento de distúrbios emocionais nessa população. Durante o internato, as angústias se intensificam. Incertezas na aplicação dos conhecimentos adquiridos, contato direto com o paciente, longos e exaustivos plantões, redução do tempo livre e a dificuldade na escolha da especialidade médica são alguns dos fatores que os estudos apontam para explicar a maior presença de sintomas de ansiedade e depressão no último ano da faculdade (Al-Faris et al., 2012; Benevides-Pereira \& Gonçalves, 2009; Costa et al., 2012; Leão, Martins, Menezes, \& Bellodi, 2011; Millan \& Arruda, 2008; Yussuf, Issa, Ajiboye, \& Buhari, 2013).

O aluno do curso de medicina encontra-se em uma jornada integral, com alto grau de exigência, necessitando internalizar vasta diversidade de conteúdos em tempo restrito, não somente referentes aos saberes acadêmicos, mas especialmente às habilidades que possibilitam as relações interpessoais, ao lidar com sentimentos, sofrimentos e a morte, envoltos em contexto de profunda competitividade, privação de lazer e insegurança técnica (Andrade et al., 2014; Bellodi, 2007; Tanaka et al., 2016). Tais características acentuam-se no ciclo clínico $\left(3^{\circ}\right.$ e $4^{\circ}$ anos), quando a frequência das atividades ambulatoriais torna o contato dos acadêmicos com os pacientes mais intenso e a carga horária de aulas é exaustiva.

De acordo com Cybulski \& Mansani (2017), a prevalência de sintomas depressivos em estudantes de medicina é preocupante no mundo inteiro, apresentando um intervalo de variação muito amplo, entre 13,9\% e $79 \%$. A mesma realidade é encontrada nas pesquisas no 
Brasil. Uma pesquisa realizada na Universidade Federal de Santa Maria (Benvegnu, Deitos, \& Copette, 1996) utilizando o Self-Reporting Questionnaire (SRQ-20) revelou que o predomínio de TMC entre os estudantes do primeiro ao décimo período do curso foi de 31,7\%. Outro estudo, conduzido na UFPE (Facundes \& Ludemir, 2005) detectou $34,1 \%$ de alunos acometidos, e na Faculdade de Medicina de Botucatu (Lima, Domingues, \& Cerqueira, 2006) a prevalência de 44,7\%.

Os principais estudos associaram TMC em estudantes de medicina aos seguintes fatores: sexo feminino; alterações no sono; não possuir transporte próprio; sedentarismo; não receber o apoio necessário; dificuldades em fazer amigos; morar sozinho; privação de lazer; contato com a morte e processos patológicos; problemas de adaptação ao local do curso; perspectivas ruins em relação ao futuro; e insatisfação com a escolha profissional (Fiorotti, Rossoni, Borges, \& Miranda, 2010; Sohail, 2013; Yussuf et al., 2013).

Diferentes estudos analisaram a prevalência de TMC em estudantes de medicina através de Self-Reporting Questionnaire (SRQ-20). Pesquisa realizada no sul do Brasil (Ferreira et al., 2016) avaliou os alunos de forma prospectiva e observou um aumento de sintomas de TMC de $35,8 \%$ no início do semestre para $51,5 \%$ ao final $(\mathrm{p}=$ 0,002). Fiorotti et al. (2010), em estudo realizado com alunos da UFES, encontraram resultados totais de 37,1\% de TCM e apontou maior prevalência entre os acadêmicos do ciclo básico (43,6\%) e do clínico (40,3\%), sendo menor entre os internos $(27 \%)(\mathrm{p}=0,025)$, notando-se os maiores índices no $2^{\circ}(52,6 \%)$ e $4^{\circ}(53,8 \%)$ anos e a menor no último ano, com $16,2 \%(\mathrm{p}=0,008)$.

Apesar da insuficiência de dados sobre o assunto, a alta prevalência de TMC encontrada em estudantes de medicina reforça a necessidade de aprofundamento do conhecimento sobre o problema, considerando a inegável importância deste. Desta forma, o presente trabalho pretende identificar a prevalência de sintomas de ansiedade e de depressão em estudantes de medicina em um curso privado do interior da região sudeste do Brasil correlacionando-a com alguns fatores socioeconômicos.

\section{Método}

O presente trabalho consiste em um estudo quantitativo epidemiológico, censitário e descritivo, por meio de questionário autoaplicável GHQ-12. O GHQ-12 (General Health Questionnaire) é uma versão resumida, contendo 12 ítens, que visa a detectar transtornos mentais comuns, com estudo de validação realizado no Brasil por Gouveia et al. (2003), sendo utilizado em estudos de diferentes áreas e com diferentes populações. Além das perguntas do GHQ, os estudantes também responderam questões que abordavam dados sócio-econômicos dos alunos,

Os dados foram coletados em sala de aula, no início ou término das aulas, pelos próprios pesquisadores, que informavam os objetivos da pesquisa. Para garantir o anonimato, os formulários eram depositados em caixas lacradas que somente foram abertas após o término da fase de coleta de dados. Posteriormente, as informações foram alimentadas em um banco de dados por meio do pacote Office 2010 e posteriormente convertidas para um banco de dados do software de análise estatística Stata 13, no qual a análise uni e bivariada foi realizada.

A pesquisa foi aprovada pelo Comitê de Ética em Pesquisa registrado com o número 51131015.2.0000.5237 e todos os participantes preencheram termo de consentimento livre e esclarecido.

\section{Resultados e discussão}

O estudo contou com a participação de 428 alunos dos seis anos do curso, perfazendo uma cobertura de $67,82 \%$ do corpo discente (631 alunos), o que representa uma excelente cobertura censitária.

Ao analisarmos a presença de sintomas depressivos nos respondentes, encontra-se uma prevalência de TMC de $58,8 \% \quad(n=252)$. As menores prevalências se concentraram nos dois primeiros anos havendo um pico no terceiro ano e uma leve diminuição até os últimos anos. Os dados de prevalência de cada ano de curso estão apresentados na Tabela 1 .

Tabela 1.

Prevalência de TMC em estudantes de medicina por ano da graduação

\begin{tabular}{lccccc}
\hline Ano & Não & $\%$ & Sim & $\%$ & Total \\
\hline 1 & 35 & $58,33 \%$ & 25 & $41,67 \%$ & 60 \\
2 & 38 & $45,78 \%$ & 45 & $54,22 \%$ & 83 \\
3 & 16 & $22,54 \%$ & 55 & $77,46 \%$ & 71 \\
4 & 27 & $44,26 \%$ & 34 & $55,74 \%$ & 61 \\
5 & 29 & $36,71 \%$ & 50 & $63,29 \%$ & 79 \\
6 & 31 & $41,89 \%$ & 43 & $58,11 \%$ & 74 \\
Total & 176 & $41,12 \%$ & 252 & $58,88 \%$ & 428 \\
\hline
\end{tabular}

Analisando-se o perfil dos acadêmicos de Medicina da instituição conclui-se que a maioria está na faixa etária entre 20 e 24 anos, totalizando $63,62 \%(n=267)$ do total. Quanto ao sexo, 66\% $(\mathrm{n}=278)$ são do sexo feminino e $81,47 \%(n=343)$ se definiram como brancos. Quanto ao estado civil, 95,73\% (n=404) eram solteiros. $\mathrm{Na}$ avaliação da religião atual $50,95 \% \quad(n=215)$ dos estudantes relataram a religião católica, seguidos de $19,19 \%(n=81)$ e $14,4 \%(n=61)$ que disseram não ter religião. No que tange a religião de criação, 81,04\% $(n=342)$ referiram ter sidos criados no catolicismo. Observou-se que a maioria dos pais possui ensino superior completo, referente a $74,9 \%(n=317)$ das mães e $64,54 \%(n=273)$ dos pais.

Os dados apontam para uma alta prevalência de TMC e para uma população extremamente homogênea, o que explica a dificuldade em se determinar associação entre as variáveis elencadas e o desfecho (TMC).

Embora os achados do presente estudo divirjam parcialmente da bibliografia disponível, este fato pode ser explicado pela maior cobertura obtida. Amostras menores estão mais sujeitas a variações aleatórias das estimativas. 


\section{Considerações finais}

A prevalência de TMC em estudantes de medicina é alta e preocupante. Os dados demonstram um aumento considerável na população afetada no decorrer do curso, indicando um possível efeito cumulativo dos fatores sociais e ambientais relacionados ao comprometimento da saúde mental dos indivíduos.

Embora não haja vínculo entre dados sócio demográficos e a presença de TMC, este fato se deve às características da população de estudo, não sendo possível afirmar categoricamente quanto a presença ou ausência de associação.

As instituições de ensino necessitam reconhecer este cenário e compreender a necessidade de enfrentamento desta preocupante condição de comprometimento da saúde de seus estudantes. Estudos que indicam esta prevalência, embora relevantes, devem ser complementados por pesquisas que procurem identificar as situações eliciadoras de sofrimento entre os acadêmicos. É fundamental fomentar ações de prevenção, mapeamento, acompanhamento e remediação que busquem melhorar a qualidade de vida no ambiente acadêmico.

Além disso, a saúde mental do estudante de medicina deve ser correlacionada com estudos que avaliam a saúde mental dos profissionais da saúde, o que poderá gerar melhorias para os profissionais e, consequentemente, para o sistema de saúde como um todo, na medida em que tais fatores influem na qualidade do atendimento e do relacionamento com os pacientes.

\section{Referencias}

Al-Faris, E. A., Irfan, F., Van der Vleuten, C. P. M., Naeem, N., Alsalem, A., Alamiri, N., ... Alofaisan, Y. (2012). The prevalence and correlates of depressive symptoms from an Arabian setting: a wake up call. Medical Teacher, 34 Suppl 1, S32-36. https://doi.org/10.3109/0142159X.2012.656755

Alves, T. C. de T. F. (2014). Depressão e ansiedade entre estudantes da área de saúde. Revista de Medicina, 93(3), 101-105.

Andrade, J. B. C. de, Sampaio, J. J. C., Farias, L. M. de, Melo, L. da P., Sousa, D. P. de, Mendonça, A. L. B. de, ... Cidrão, I. S. M. (2014). Contexto de formação e sofrimento psíquico de estudantes de medicina. Revista Brasileira de Educação Médica, 38(2), 231242.

Bellodi, P. L. (2007). Retaguarda emocional para o aluno de medicina da Santa Casa de São Paulo (REPAM): realizações e reflexões. Rev. bras. educ. med, 31(1), 514.

Benevides-Pereira, A. M. T., \& Gonçalves, M. B. (2009). Transtornos emocionais e a formação em Medicina: um estudo longitudinal. Revista Brasileira de Educação Médica, 33(1), 10-23. https://doi.org/10.1590/S0100-55022009000100003

Benvegnu, L., Deitos, F., \& Copette, F. (1996). Problemas psiquiátricos menores em estudantes de Medicina da Universidade Federal de Santa Maria, RS, Brasil. Rev Psiquiatr Rio Gd Sul, 18, 229-233.
Costa, E. F. de O., Santana, Y. S., de Abreu Santos, A. T. R., Martins, L. A. N., de Melo, E. V., \& de Andrade, T. M. (2012). Sintomas depressivos entre internos de medicina em uma universidade pública brasileira. Revista da Associação Médica Brasileira, 58(1), 5359.

Cunha, M. A. B., Neves, A. A. de F., Moreira, M. E., Hehn, F. J., Lopes, T. P., Ribeiro, C. C. F., \& Watanabe, Á. de P. F. (2009). Minor psychiatric disorders and the search for care by medical students. Revista Brasileira de Educação Médica, 33(3), 321328.

Cybulski, C. A., Mansani, F. P., Cybulski, C. A., \& Mansani, F. P. (2017). Análise da Depressão, dos Fatores de Risco para Sintomas Depressivos e do Uso de Antidepressivos entre Acadêmicos do Curso de Medicina da Universidade Estadual de Ponta Grossa. Revista Brasileira de Educação Médica, 41(1), 92101.

https://doi.org/10.1590/1981-52712015v41n1rb20160 034

Facundes, V., \& Ludemir, A. B. (2005). Transtornos mentais comuns em estudantes da área de saúde. Rev Bras Psiquiatr., 27(3), 194-200.

Ferreira, C. M. G., Kluthcovsky, A. C. G. C., \& Cordeiro, T. M. G. (2016). Prevalência de Transtornos Mentais Comuns e Fatores Associados em Estudantes de Medicina: um Estudo Comparativo. Revista Brasileira de Educação Médica, 40(2), 268-277. https://doi.org/10.1590/1981-52712015v40n2e028120 14

Fiorotti, K. P., Rossoni, R. R., Borges, L. H., \& Miranda, A. E. (2010). Transtornos mentais comuns entre os estudantes do curso de medicina: prevalência e fatores associados. J Bras Psiquiatr, 59(1), 17-23.

Leão, P. B. de O. e S., Martins, L. A. N., Menezes, P. R., \& Bellodi, P. L. (2011). Well-being and help-seeking: an exploratory study among final-year medical students. Revista da Associação Médica Brasileira, 57(4), 379-386. https://doi.org/10.1590/S0104-42302011000400009

LIMA, M. C. P., DOMINGUES, M., \& CERQUEIRA, A. (2006). Prevalência e fatores de risco para transtornos mentais comuns entre estudantes de medicina. Rev Saúde Pública, 40(6), 1035-1041.

Millan, L. R., \& Arruda, P. C. V. de. (2008). Assistência psicológica ao estudante de medicina: 21 anos de experiência. Revista da Associação Médica Brasileira, 54(1), 90-94. https://doi.org/10.1590/S0104-42302008000100027

Pereira, N. K. C., Padoim, I., \& Junior, R. F. (2014). Psychosocial and health-related stressors faced by undergraduate medical students. Revista de Medicina, 93(3), 125-134.

Rocha, E. S., \& Sassi, A. P. (2013). Minor mental disorders among medical students. Revista Brasileira de Educação Médica, 37(2), 210-216.

Santa, N. D., \& Cantilino, A. (2016). Suicídio entre Médicos e Estudantes de Medicina: Revisão de Literatura. Revista Brasileira de Educação Médica, 40(4),

$772-780$ 
https://doi.org/10.1590/1981-52712015v40n4e002620 15

Sohail, N. (2013). Stress and academic performance among medical students. Journal of the College of Physicians and Surgeons--Pakistan: JCPSP, 23(1), 67-71. https://doi.org/01.2013/JCPSP.6771

Tanaka, M. M., Furlan, L. L., Branco, L. M., \& Valerio, N. I. (2016). Adaptação de Alunos de Medicina em Anos Iniciais da Formação. Revista Brasileira de Educação Médica, 40(4), 663-668. https://doi.org/10.1590/1981-52712015v40n4e006920 15

World Health Organization, Victorian Health Promotion Foundation, \& University of Melbourne. (2004). Promoting mental health: concepts, emerging evidence, practice : summary report. Geneva: World Health Organization. Recuperado de http://www.myilibrary.com?id=9728

Yussuf, A. D., Issa, B. A., Ajiboye, P. O., \& Buhari, O. I. (2013). The correlates of stress, coping styles and psychiatric morbidity in the first year of medical education at a Nigerian University. African Journal of Psychiatry, 16(3), 206-215. https://doi.org/http://dx.doi.org/10.4314/ajpsy.v16i3.2 8 